\title{
Influencia de la gastronomía mexicana en el consumo étnico en España
}

\section{Influence of Mexican gastronomy on ethnic consumption in Spain}

Ramón Sebastián Acle M. ${ }^{1}$, Aylin Vanessa Montiel A. ${ }^{2}$

Resumen: La gastronomía es un arte conformado por el conjunto de conocimientos y actividades relacionadas con la elaboración de los alimentos que pertenecen a la cultura de una sociedad. Su importancia radica en que refleja las costumbres, la tradición, la historia, la geografía y el estilo de vida del lugar al que pertenece. Debido al gran impacto que ha tenido la gastronomía mexicana en España, es de gran interés profundizar en su consumo, que se refleja en un sinnúmero de restaurantes, tiendas y supermercados que se encargan de llevar al mercado español productos mexicanos, también conocidos como productos étnicos. El objetivo principal de este trabajo es medir la influencia que tiene la gastronomía mexicana en el consumo étnico en España. La presente investigación muestra un marco teórico que permitió la explicación de los términos gastronomía mexicana, producto étnico y España. Se diseñó una metodología cuantitativa a través del modelo de las 4 P's de McCarthy (producto, precio, plaza y promoción) y su relación con la gastronomía mexicana en el consumo étnico en España. Se utilizaron los métodos; deductivo, sintético y analítico que permitieron evaluar la correlación de las variables. Los resultados mostraron evidencia a favor del consumo de los productos étnicos.

Palabras clave: Gastronomía mexicana, productos étnicos, metodología cuantitativa.

\begin{abstract}
Gastronomy is an art comprised of the knowledge and activities related to preparing the foods that belong to the culture of a society. Its importance lies in its reflection of the customs, tradition, history, geography and lifestyle of the place to which it belongs. Given the tremendous impact that Mexican gastronomy has had on Spain, it is of great interest to delve more deeply into its consumption, which is reflected in an endless number of restaurants, shops and supermarkets bringing Mexican products, also known as ethnic products, to the Spanish market. The primary aim of this study is to gauge the influence that Mexican gastronomy has on ethnic consumption in Spain. This study presents a theoretical framework to explain the terms Mexican gastronomy, ethnic product and Spain. A quantitative methodology was designed using the model of McCarthy's 4 Ps (product, price, place and promotion) and its relation to Mexican gastronomy in ethnic consumption in Spain. The methods used were: deductive, synthetic and analytical, which enabled an assessment of the correlation of the variables. The results showed evidence in favor of the consumption of ethnic products.
\end{abstract}

Key words: Mexican gastronomy, ethnic products, quantitative methodology.

\section{(Presentado: 19 de noviembre de 2017. Aceptado: 3 de enero de 2018)}

\footnotetext{
Doctor en Administración Gerencial. Benemérita Universidad Autónoma de Puebla, Facultad de Administración. Av. San Claudio Sn, Cd Universitaria, San Manuel, 72570 Puebla, México. E-mail: raclemxayahoo.com

2 Licenciatura en Administración de Empresas. Benemérita Universidad Autónoma de Puebla. E-mail: aylinmontađggmail.com
} 


\section{INTRODUCCIÓN}

Los productos étnicos son aquellos asociados a un país, pero que en el exterior los consumen tanto los nacionales como otros grupos de población (CEPAL, 2004), éstos engloban tanto a: artesanías, juguetes, dulces típicos, música y ropa, así como alimentos y bebidas que son los productos con más demanda en otros mercados internacionales, estos productos forman parte intrínseca de la cultura gastronómica de un país. El desarrollo de flujos comerciales y migratorios, así como la mejora de los medios de comunicación y transporte, han jugado un papel determinante en la formación y desarrollo del mercado de este tipo de alimentos en países ajenos del que provienen originalmente.

Actualmente, la cocina mexicana se está distinguiendo de las del resto del mundo por sus tradicionales recetas, el sabor, la calidad y el uso abundante de especias. En el 2010 la UNESCO, reconoció a la gastronomía mexicana como Patrimonio Inmaterial de la Humanidad, ya que es única y distingue al país de otros, desde la cosecha de alimentos hasta la forma de elaboración de sus diversos platillos (Mondragón, 2015). La gastronomía mexicana está presente en diversas partes del mundo, principalmente en Estados Unidos, España, China y Francia, entre otros. Debido al gran impacto que ha tenido la gastronomía mexicana en el mundo pero, principalmente, en España, es de gran interés profundizar en su consumo, ya que como consecuencia hay un sinfín de restaurantes, tiendas, supermercados y demás, que se encargan de llevar al mercado español productos mexicanos, también conocidos como productos étnicos.

Por último, el estudio se encuentra organizado de tal forma que para la revisión literaria se describen conceptos de: gastronomía mexicana, producto étnico y España, siendo palabras clave para el correcto desarrollo del tema. Posteriormente se describe la metodología utilizada y por último los resultados obtenidos, conclusiones y referencias.

\section{Gastronomía mexicana}

La cultura es todo aquello que constituye a un país y configura su identidad, ya que refleja el estilo de vida, costumbres y tradiciones, las cuáles son transmitidas de una generación a otra, presentando características de la sociedad donde se originan. La cultura engloba desde la vestimenta de las personas que habitan en una determinada región hasta la forma de preparación de sus alimentos (Giménez, 2007).

Por lo tanto, la comida es cultura cuando se produce, se prepara y se consume. Son los tres momentos más importantes de un proceso vital, el alimentarse, pero no nada más por cubrir la necesidad fisiológica del comer, sino por su papel en el desarrollo de la historia, identidad y cultura del ser humano (Montanari, 2006). Es por ello, que la cocina juega un lugar esencial en la cultura, ya que a través de ésta se representan los sabores, gustos, costumbres, tradiciones y valores, los cuales se ven reflejados al momento de preparar un alimento, en los utensilios de cocina con sus significados y funciones de acuerdo al entorno, en la organización y motivo para su elaboración, y en las distintas costumbres y tradiciones que lo acompañan. En otras palabras, la gastronomía es cultura.

La gastronomía deriva del griego "gaster" o "gastros" que significa estómago y "gnomos", conocimiento o ley, es decir, la gastronomía es el conocimiento razonado, entendimiento, sobre todo lo que tiene que ver con la comida (Muzeum Gastronomie, 2018), y su relación con el hombre a lo que su alimentación se refiere (Alva, 2012). Frecuentemente se piensa que el término "gastronomía" únicamente tiene relación con el arte de cocinar y la diversidad de platillos, sin embargo, esta es una pequeña parte, ya que se vincula también con: a) la historia, en lo que se refiere a la clasificación de los insumos, b) el comercio, por la búsqueda de formas de compra que permiten al consumidor adquirir la mercancía con las mejores ventajas de calidad y precio, c) la cocina, al momento de preparar los alimentos y convertirlos en algo agradable para el paladar, y d) Las costumbres y tradiciones de cada región.

Es por esto, que la importancia de la gastronomía radica en que muestra la cultura de cada país al que pertenece, haciendo énfasis en la forma de vida, antigüedad, aprovechamiento de los recursos locales y la evolución de cada región del mundo. Otro atributo relevante de la gastronomía actual es su poder para atraer al turismo, una prueba de esto es que hay un gran número de personas que deciden viajar realizando rutas gastronómicas por el mundo; éstas se centran en los sabores típicos de cada región.

Actualmente la gastronomía cuenta con una gran variedad de platillos, abarcando aspectos muy importantes que dependen del tipo de cocina de la que se esté hablando, ya que cada una muestra su origen, evolución, cultura y tradiciones (Alvarado, 2011).

Dentro de los tipos de gastronomía se encuentra la internacional, que abarca las cocinas de diferentes partes del mundo. Entre las más importantes están: la dieta mediterránea, la cocina mexicana, la francesa y la japonesa, 
que incluso atraen el turismo por sus peculiares platillos ( National Geographic, 2015), es por ello que en el 2010 con el fin de reconocer el valor cultural de la cocina, la Organización de las Naciones Unidas para la Educación, la Ciencia y la Cultura (UNESCO), reconoció a estas cuatro gastronomías como Patrimonio Inmaterial de la Humanidad (Aparicio, 2014). Asimismo, la gastronomía nacional es aquella que se considera propia y cotidiana de un país, no es considerada como una expresión de individualidad regional, sino más bien, como un aspecto de la identidad grupal (Torres, Madrid de Zito Fontán, \& Santoni , 2004).

La gastronomía mexicana actualmente es considerada elemento crucial de identidad nacional, debido a su historia, creatividad, diversidad y trascendencia. También goza de un papel importante como elemento de identidad para el pueblo mexicano, es de elaboración colectiva, se apoya en productos originarios de su tierra y presume una gran creatividad en sus cocineros, mismos que a lo largo de los siglos han ido perfeccionando esta gastronomía. Y es que la cocina mexicana además de ser todo eso, se encarga de combinar técnicas contemporáneas que le otorgan el carácter de innovación y adaptación constante. Gracias a esto, ha logrado conservarse y permanecer a lo largo de varias generaciones (Fernández, 2016).

El secreto de la gastronomía mexicana es la mezcla de las distintas cocinas que conviven en su territorio, empezando por la de sus antiguos habitantes, mayas y aztecas así como de la influencia de la comida española y norteamericana, y el uso de productos autóctonos, sobre todo la gran variedad de chiles que define el sabor de sus platillos (National Geographic, 2015).

Por otra parte, México utiliza en la preparación de sus alimentos, ingredientes tan variados como la cebolla morada, el aguacate, los chiles rojos, los pimientos amarillos y los piñones. A pesar de esta gran diversidad mantiene una línea común, un ingrediente que aparece en casi todos los platillos es el maíz, protagonista en la elaboración de tortillas, bebidas fermentadas, atole, tamales y pozole, entre otros alimentos (FORBES, 2014).

Cada región del país cuenta con alimentos representativos que muestran su cultura y estilo de vida. En la Tabla 1 se presentan las gastronomías nacionales más representativas de México.

\section{Tabla 1. Gastronomías nacionales más representativas de México.}

\begin{tabular}{|c|c|c|}
\hline $\begin{array}{l}\text { Regiones } \\
1\end{array}$ & $\begin{array}{l}\text { Estados } \\
\text { Baja California Sur, Baja California } \\
\text { Norte, Sonora, Chihuahua, Coahuila, } \\
\text { Nuevo León }\end{array}$ & $\begin{array}{l}\text { Platillos } \\
\text { Sopa de aleta de tiburón, cabrito relleno, carne } \\
\text { asada, chiles rellenos de mariscos, marones rellenos } \\
\text { de queso, machaca, tortillas de harina. }\end{array}$ \\
\hline 2 & $\begin{array}{l}\text { Sinaloa, Nayarit, Jalisco, } \\
\text { Aguascalientes, Zacatecas, Durango. }\end{array}$ & $\begin{array}{l}\text { Pescado zarandeado, pipián con papas, pozole rojo, } \\
\text { tortas ahogadas, frijoles puercos, sopa de tortilla. }\end{array}$ \\
\hline 3 & $\begin{array}{l}\text { Colima, Michoacán, Guerrero, Veracruz, } \\
\text { Tamaulipas, Tabasco. }\end{array}$ & $\begin{array}{l}\text { Ceviche preparado con carne molida de pez, el caldo michi, } \\
\text { cecina, jaiba en chilpachole y arroz blanco con plátanos } \\
\text { machos, guisado con pescado blanco y chalupas. }\end{array}$ \\
\hline 4 & Chiapas, Oaxaca & $\begin{array}{l}\text { Cochinita al horno, tamales de bola y chipilín, chalupas, pozol } \\
\text { blanco, pozol de cacao, pan de elote, mole, chilatole, } \\
\text { chapulines y tlayudas. }\end{array}$ \\
\hline 5 & $\begin{array}{l}\text { Estado de México, Tlaxcala, Distrito } \\
\text { Federal, Puebla, Morelos. }\end{array}$ & $\begin{array}{l}\text { Quesadillas, tostadas, tlacoyos, huaraches, mixiote, } \\
\text { cecina con guacamole y gusanos de maguey. }\end{array}$ \\
\hline 6 & $\begin{array}{l}\text { Querétaro, San Luis Potosí, } \\
\text { Hidalgo, Guanajuato. }\end{array}$ & $\begin{array}{l}\text { Empanadas de carnitas, pico de gallo, enchiladas } \\
\text { mineras, caldo michi, escamoles, chimicuiles y chilaquiles. }\end{array}$ \\
\hline 7 & Campeche, Yucatán, Quintana Roo. & $\begin{array}{l}\text { Pejelagarto, sopa de mariscos, venado, tortuga, langosta } \\
\text { en su jugo, cochinita pibil con escabeche, y sopa de lima. }\end{array}$ \\
\hline
\end{tabular}

Fuente: Elaborado en base a Hinojosa (2011) 
Cabe mencionar que los ingredientes gastronómicos que más destacan en cada una de las regiones del país son: maíz, chile y frijol, los cuáles definen la esencia gastronómica nacional. El origen del maíz, con toda probabilidad, fue en América Central, por lo cual para las civilizaciones maya y azteca tuvo un importante papel en sus creencias religiosas, festividades y nutrición. Actualmente México es el $7^{\circ}$ lugar como productor a nivel mundial. Entre los alimentos preparados con maíz se puede destacar; la tortilla, chilaquiles, elotes, huitlacoche, pinole, tamales y tacos, entre otros (Compartido - Fideicomiso de Riesgo, 2017). El chile es el sello distintivo de la cocina mexicana y continúa siendo un ingrediente imprescindible en cualquier mesa. Se puede preparar tanto en caldos para enchiladas verdes o adobos, como en ricas salsas que condimentan carnes, tacos y otros guisos. Se consumen desde las variedades más suaves y dulces, como los coloridos pimientos, hasta las que necesitan un litro de agua para calmar el picor, como los habaneros, los serranos y el piquín. Por último, el frijol es otro alimento primordial de la dieta del mexicano. Está presente en la mayoría de los antojitos, como los sopes, las tlayudas oaxaqueñas y los panuchos de Yucatán. También se pueden saborear los frijoles de olla cocidos con una aromática rama de epazote.

De acuerdo con la Secretaría de Agricultura, Ganadería, Desarrollo Rural, Pesca y Alimentación (SAGARPA), los productos de exportación más representativos de México para el mundo, gracias a su variedad y calidad son: a) Jackfruit: Es el fruto del árbol conocido como "jaca", "Jack" o "yaca", contiene una pulpa de color amarillo de sabor agridulce. Aunque es originario de Indonesia, Nayarit es líder en producción anual; b) La confitería: Se ubica junto con otros productos en el $4^{\circ}$ lugar de exportación con derivados como chicle natural, fruta deshidratada, rollos de fruta, cereales, salsas, snacks (botanas), chocolates, caramelos, pastillas de caramelo macizo, dulces mexicanos de leche, paletas de caramelo, entre otros; c) Nopalitos: Esta verdura rica en fibra, goza de una enorme versatilidad para su preparación, pues se puede consumir crudo, asado o guisado, o bien en mermeladas, dulces regionales y como producto cosmético; d) Amaranto: Su uso más conocido es en dulces mexicanos como las alegrías, pero también se emplea reventado en granola y harinas. Los principales destinos de exportación son Italia, Chile y EE.UU.; e) Coliflor: Se usa comúnmente en la preparación de sopas, guisos, ensaladas, y también en postres y f) Guayaba: Esta fruta deleita paladares de España, Reino Unido, Canadá y Estados Unidos.
Asimismo, SAGARPA en el 2017 reporto los principales destinos de las exportaciones de alimentos y bebidas mexicanas siendo Estados Unidos, Japón, Canadá y Guatemala. Cabe destacar que China actualmente representa un mercado potencial para estos productos.

Los productos agropecuarios más apreciados y exportados al extranjero son: a) Cerveza: siendo la bebida de mayor exportación y considerando a México el cuarto productor a nivel mundial, exportándose principalmente a Estados Unidos, Canadá, Chile y Australia, b) Aguacate: es el segundo producto del campo más exportado siendo México el productor número uno del mundo, su mayor demanda es Estados Unidos, así como Canadá, Japón, Países Bajos, Francia, El Salvador y China, c) Jitomate: se exporta principalmente a Estados Unidos, siendo México el décimo productor a nivel mundial, d) Tequila: es la bebida alcohólica mexicana con denominación de origen más exportada, los principales países consumidores de tequila en el extranjero son Estados Unidos y Francia, e) Carne de res: a nivel mundial, México es el sexto país productor con mayor potencial en Estados Unidos, Italia, Países Bajos, Francia, Alemania y Rusia y f) Berries: comprendiendo al arándano, fresa, frambuesa, grosella y zarzamora y colocando a México en el sexto lugar en materia de exportación (CNN, 2018).

Después de todo lo expuesto anteriormente, se puede concluir que la cocina mexicana es una de las más importantes en el mundo, ha conquistado diversos países, gracias a la variedad de sus platillos y sabores, sus costumbres, tradiciones y su peculiaridad, entre estos España, que últimamente ha incrementado la apertura de restaurantes cuya especialidad es la comida mexicana, además de que ha considerado alimentos mexicanos como productos étnicos, tales como las salsas, el mole poblano, los tacos y el aguacate, entre otros.

\section{Productos étnicos}

Un producto es un conjunto de atribuciones tangibles (bienes) e intangibles (servicios) que incluye el empaque, color, precio, prestigio del fabricante y del vendedor, que el comprador puede aceptar como algo que ofrece satisfacción a sus necesidades o deseos (Stanton, Etzel, \& Walker, 2007).

Los productos se pueden clasificar en bienes de consumo: aquellos destinados a satisfacer directamente las necesidades humanas y los bienes industriales: todos los que se 
utilizan principalmente para la fabricación de otros bienes (Araujo, 2007). Dentro de los bienes de consumo se encuentran los productos étnicos, que son los que pertenecen a un país, pero que en el exterior los consumen tanto los nacionales como otros grupos de población (CEPAL, 2004). Es decir, los productos étnicos han logrado expandir sus mercados a otras dimensiones. Los productos étnicos engloban tanto artesanías, juguetes, dulces típicos, música y ropa, así como alimentos y bebidas que son los productos con más demanda en otros mercados internacionales. Sin embargo, las personas suelen no saber diferenciar los productos étnicos de los de nostalgia, ambos son provenientes de sus respectivas naciones, pero los de nostalgia se consumen por un grupo determinado de personas que emigran al extranjero, quienes generalmente extrañan estos productos, los cuales son difíciles de conseguir en los nuevos lugares a los que se mueven y los étnicos son aquellos asociados a un país, pero que en el exterior los consumen tanto los nacionales como otros grupos de población (CEPAL, 2004). Los productos étnicos deben cumplir con normas sanitarias y fitosanitarias para que puedan abarcar otras fronteras como una estandarización a escala internacional en cuanto a normas de calidad.

Según el estudio realizado por la Comisión Económica para América Latina y el Caribe (CEPAL), una de las virtudes que poseen los productos étnicos, es que se elaboran de forma artesanal, tal es el caso del tequila o del guacamole, los cuales por su calidad logran cumplir los estándares para poder ubicarse en el extranjero (CEPAL, 2004). Los alimentos étnicos forman parte intrínseca de la cultura gastronómica de un país. El desarrollo de flujos comerciales y migratorios, así como la mejora de los medios de comunicación y transporte, han jugado un papel determinante en la formación y desarrollo del mercado de este tipo de alimentos en países ajenos del que provienen originalmente. Para el caso de México, los productos étnicos han logrado posicionar a la gastronomía mexicana como una de las mejores del mundo. También han sido una oportunidad de mercado para empresas que se dedican a exportar estos productos a otros países. Dentro de los alimentos más demandados se encuentra el chile que es considerado como uno de los más representativos, además de que forma parte de la dieta mexicana conformada también por el maíz y frijol.

Productos como el tomate, aguacate, tequila y chile, entre otros, se encuentran entre los primeros lugares de consumo a nivel mundial; el clima y los costos bajos han permiti- do el alto posicionamiento de éstos. De esta forma, México es el principal país exportador del sector agroalimentario del mundo (Chávez, 2014). En la tabla 2 se muestran los 10 productos étnicos pertenecientes a México con mayor exportación y demanda en el extranjero (Tabla 2).

\section{Tabla 2. Los 10 productos étnicos pertene- cientes a México más demandados en el ex- tranjero.}

\begin{tabular}{cc}
\hline Lugar de exportación & Producto \\
1 & Cerveza de malta \\
2 & Tomate \\
3 & Aguacate \\
4 & Tequila \\
5 & Azúcar de caña \\
6 & Berries \\
7 & Confitería \\
8 & Chile bell \\
9 & Pepinos \\
10 & Demás frutos \\
\hline
\end{tabular}

Fuente: Elaborado en base a El Financiero (2014).

La gastronomía mexicana ha conquistado diversas partes del mundo, tal es el caso específico de España, ya que la inmigración ha sido un elemento decisivo en la comercialización de los alimentos étnicos. A principios de los noventa, cuando la población inmigrante representaba tan sólo $0.9 \%$ de la población total, los niveles de demanda hacia alimentos y cocinas específicamente extranjeras era reducida (Camarena \& Sanjuán, 2007). La comida mexicana es la segunda con más ventas en el mercado español después de la china. Algunas estimaciones la sitúan entre las de mayor potencial de crecimiento en el mercado español previendo un crecimiento de ventas a un ritmo de $18.6 \%$ anual. En un estudio de mercado de la comida étnica en España de Camarena y Sanjuan en el 2008, aseguran que las causas del éxito de la comida mexicana no se relacionan directamente con los flujos migratorios, ya que contrariamente a lo que sucede en otros países como Estados Unidos, donde los mexicanos conforman un grupo de inmigrantes mayoritario, en España, el número de inmigrantes mexicanos se encuentra muy alejado de otras nacionalidades, como los ecuatorianos y marroquíes. En consecuencia, la comida étnica mexicana no se sustenta en el consumo que de los inmigrantes procedentes de ese país, sino que son otros segmentos los que en buena medida realizan la mayor demanda de estos alimentos (Camarena \& Sanjuán, 2007). 
En la atualidad los alimentos étnicos son consumidos y demandados, tanto por parte de la población extranjera como por la nativa. No obstante, las motivaciones que llevan a cada segmento de consumidores a degustarlos son distintas. En el caso de los inmigrantes, el consumo de alimentos étnicos se relaciona con la continuidad de sus tradiciones, los sentimientos hacia su país de origen, la búsqueda de emociones, el resguardo de su identidad, los recuerdos de su familia y la confianza que sienten hacia productos que ya conocen y con los cuales están familiarizados (Cruz, López , \& Schatan, 2004). Los consumidores nacionales, por su parte, se aproximan a los alimentos étnicos buscando conocer aspectos gastronómicos de otras culturas, motivados por la curiosidad y el deseo de probar sensaciones y sabores nuevos, así como la evocación de sabores y aromas que les trasladen a experiencias pasadas o a futuros destinos exóticos (Camarena \& Sanjuán, 2007). En los últimos años, el conocimiento y consumo de las comidas y/o alimentos étnicos se ha difundido internacionalmente. En España, los alimentos étnicos comenzaron a comercializarse en la década de los años noventa, sin embargo, el mayor auge de sus ventas se ha producido a partir del año dos mil y se han constituido una de las gamas de alimentos con más potencial de expansión.

En conclusión, la cocina mexicana caracterizada por una gran variedad de platillos y recetas, así como la complejidad de su elaboración está tomando una enorme importancia y reconocimiento, y los ojos de todo el mundo están volteando a los platillos mexicanos, ya que forma parte de un legado de un patrimonio riquísimo de miles de años de sabores distintivos y condimentados, reuniendo tradiciones gastronómicas indígenas y europeas, entre otras muchas. Actualmente la gastronomía mexicana se fusiona de manera armónica entre la comida tradicional y de vanguardia, donde una nueva ola de jóvenes cocineros y chefs están poniendo el nombre de México en alto en restaurantes alrededor del mundo. Por último, los productos étnicos han trascendido fronteras, en particular, los productos originarios de México, no solamente los alimentos, sino también juguetes, artesanías y ropa. Uno de los mercados a dónde han logrado expandirse es el mercado español, que cuenta con un gran número de restaurantes de comida mexicana y locales dónde se comercializan productos originarios de México. Sin duda alguna, la exportación de estos productos a España es una gran oportunidad para las empresas mexicanas.

\section{España}

España se ha convertido en uno de los países más importantes del mundo debido a que cuenta con un inigualable legado histórico, infraestructuras turísticas inmejorables, gran diversidad cultural, múltiples paisajes y clima envidiable, entre otros aspectos que hacen que sea uno de los mejores lugares para vivir y visitar (Skyscanner, 2016). Además de ser un país de competitivo en la Unión Europea, moderno e innovador, dotado de recursos humanos y tecnológicos y con un gran mercado internacional. Este país se caracteriza por un alto nivel de bienestar y salud, según el Índice de Progreso Social (IPS), esta posición se debe a las altas puntuaciones obtenidas en tres categorías en particular: la salud y el bienestar, donde ocupa el primer puesto del mundo, la calidad medioambiental y el acceso a los conocimientos básicos, donde logra la cuarta posición mundial (La Vanguardia. 2017).

De acuerdo a lo anterior, la sociedad española se sitúa en el puesto décimo tercero en el ránking de países con mejor imagen exterior, de acuerdo con los medidores internacionales. Su reputación la conforman tres variables: la calidad de vida, la calidad institucional y el nivel de desarrollo. La calidad de vida española se conforma por la gente amable, el entorno cultural, el estilo de vida, el ocio y entretenimiento. Por otro lado, la calidad institucional se obtiene mediante la seguridad, el bienestar social, el respeto internacional y el entorno económico y político. Y por último el nivel de desarrollo, conformado por la gente educada, la cultura, la calidad de productos, el sistema educativo, las empresas reconocidas y la tecnología e innovación (Cruz, 2017). Dentro del nivel de desarrollo, el mercado español es uno de los más grandes de Europa, con 47 millones de consumidores, a los que hay que sumar los 56 millones de turistas que visitan España todos los años. Es por esto que España tiene alrededor de 13,000 empresas extranjeras. Todas ellas se benefician del clima de negocios atractivo y un sistema tributario que incentiva la inversión.

España es uno de los países europeos con mayor extensión territorial, tiene una superficie de $505,990 \mathrm{~km}^{2}$ y está organizado territorialmente en 17 comunidades autónomas y 50 provincias. Según estudios realizados por el Instituto Nacional de Estadística (INE) en el 2011, España cuenta con una población de 46, 815,916 habitantes (CIA, 2018). Dentro de las comunidades más importantes se encuentra Madrid, con una población de 6.5 millones de personas, siendo esta una de las provincias que más des- 
tacan, ya que además de ser la capital, es uno de los destinos turísticos más visitados en el mundo (LA VANGUARDIA, 2017). Pero también es uno de los lugares con un amplio mercado y dónde más se concentra el comercio. Actualmente, el mercado español se ha interesado por los productos mexicanos, particularmente, por la gastronomía mexicana, tan es así que en los últimos años un gran número de restaurantes de comida mexicana han abierto sus puertas a los consumidores nacionales y extranjeros. Siendo Madrid el destino dónde se concentran el mayor número de comercios dedicados a la venta de productos mexicanos, considerados productos étnicos, que como ya se ha mencionado anteriormente, son alimentos que pertenecen a una cultura específica pero cuyo consumo se extiende fuera de sus fronteras (CEPAL, 2004).

Después de todo lo planteado, sin duda Madrid es la opción más viable para realizar la presente investigación, ya que según un informe de la Organización Mundial del Turismo (OMT) España es el tercer lugar en el mundo que más turistas recibe y también es uno de los países con una reputación casi excelente, conformada por su calidad de vida, calidad institucional y su nivel de desarrollo (OMT, 2016). Por último, Madrid es el destino español que cuenta con una variedad de restaurantes de comida mexicana y con más cantidad de comercios que se dedican a la venta de productos mexicanos, lo que facilita el presente estudio de investigación.

\section{METODOLOGÍA}

La investigación preliminar fue realizado en un contexto mercadológico - cultural, bajo un enfoque mixto (cuantitativa y cualitativa). El objetivo general fue determinar la influencia que tiene la gastronomía mexicana en el consumo étnico en Madrid, España. En cuanto a la hipótesis general fue de tipo correlacional siendo enunciada; "La influencia de la gastronomía mexicana está determinada por el consumo de productos étnicos por parte de los españoles en Madrid, España”. Se utilizó el tipo de investigación correlacional para la parte cuantitativa y la descriptiva para la cualitativa. Los método utilizados fueron el deductivo, analítico y sintético.

Se utilizaron las técnicas de investigación encuesta y entrevista, utilizando el cuestionario como instrumento de medición, cuyo contenido fue de 24 ítems, utilizando la escala Likert con opción de respuesta cerrada: totalmente de acuerdo (5), de acuerdo (4), neutral (3), en desacuerdo
(2) y totalmente en desacuerdo (1), además de cuatro preguntas descriptivas demográficas (género, edad, lugar de procedencia, nivel de estudios y ocupación). La encuesta se aplicó a través de una entrevista cara a cara durante el mes de marzo de 2018 en los siguientes sitios públicos (universidades, restaurantes, y centros comerciales). La unidad de análisis fue la ciudad de Madrid, los sujetos de estudio fueron ciudadanos de Madrid con un rango de edad entre los 20 y 60 años de edad, cuyo universo fue de 1850000 ciudadanos. Para determinar el tamaño de la muestra, se utilizó la fórmula bajo los criterios de $95 \%$ de confiablidad, $5 \%$ de margen de error, $50 \%$ de probabilidad a favor y $50 \%$ de probabilidad en contra, resultando 384 encuestados de acuerdo a la siguiente fórmula:

$$
n=\frac{N z_{\alpha}^{2} p(1-p)}{e^{2}(N-1)+z_{\alpha}^{2} p(1-p)}
$$

En cuanto a las pruebas estadísticas se utilizaron el coeficiente de alfa de Cronbach para medir la confiabilidad del instrumento de medición (cuestionario) obteniéndose un valor de 0.85 cuyo significado es bueno para su aplicación. Además se utilizó el coeficiente de correlación de Pearson para medir la relación entre las variables y comprobar la hipótesis. Los programas estadísticos utilizados para realizar las operaciones estadísticas fueron Minitab y Excel.

El modelo utilizado para las variables independientes fue el Modelo Marketing Mix que tiene sus orígenes en los años 60, cuando Neil Borden identificó doce elementos de marketing controlables que gestionados adecuadamente, darían lugar a una "Operación comercial rentable". Unos años después Jerome McCarthy redujo los 12 factores propuestos por Borden a un marco simple de cuatro elementos: producto, precio, plaza y promoción (Constantinides, 2006). Se justifica la utilización de este modelo ya que sigue siendo el más utilizado para realizar las funciones del marketing, así como de seguir siendo el más enseñado en las escuelas de negocios en todo el mundo.

A continuación en la Tabla 3 se describen los dos modelos; Marketing Mix de Borden y las 4 P's de McCarthy y en la Tabla 4 las variables que miden el consumo étnico que se utilizaron para la elaboración de un constructo más viable para determinar la influencia de la gastronomía mexicana en el consumo étnico. 
Tabla 3. Modelo de Marketing Mix de Borden y las 4 P's de McCarthy.

\begin{tabular}{|c|c|}
\hline Variable & Características \\
\hline \multirow{4}{*}{ Producto } & Calidad: materiales, diseño y el método de fabricación. \\
\hline & Presentación: modelos y tamaños disponibles. \\
\hline & Envoltura: cualidades de protección, atractivo, color y redacción. \\
\hline & $\begin{array}{l}\text { Marca: adecuada con respecto al valor de la memoria, agradable, aceptable y con posibilidad } \\
\text { de expansión para poder incluir otros productos en la línea. }\end{array}$ \\
\hline Precio & Dentro del país, en el extranjero, descuentos, demanda de un producto y precios a minoristas y mayoristas. \\
\hline Plaza & $\begin{array}{l}\text { Métodos eficientes de transportación, clasificación de mercancías, técnicas para asegurarse de tener } \\
\text { una existencia adecuada de bienes accesibles en el momento y el sitio adecuado, canales de } \\
\text { distribución y colocación de productos. }\end{array}$ \\
\hline Promoción & Ventas personales, publicidad, exhibiciones, propaganda y relaciones públicas. \\
\hline
\end{tabular}

Fuente: Elaboración propia con base en la información obtenida en la investigación documental.

Tabla 4. Variables que miden el consumo étnico.

\section{Variables}

Conocimiento de los alimentos étnicos

A través de restaurantes especializados en España.

A través de viajes a otros países.

A través de conocidos y amigos de ese país.

A través de amigos.

A través de supermercados e hipermercados.

Situación de consumo
Para preparar algo rápido.

Por gusto de la marca.

Por hábitos de compra.

Fuente: (Camarena \& Sanjuán, 2007).

Para fines más exactos, se combinaron el modelo marketing mix de Borden y de las 4 P's McCarthy, así como las variables que miden el consumo étnico, para crear el siguiente constructo que tiene como objetivo conceptua- lizar las variables independientes (producto, precio, plaza y promoción), formular sus ítems y determinar la escala Likert que servirán para la construcción del instrumento de medición (cuestionario) (Tabla 5). 
Tabla 5. Constructo para validar conceptualizar metodológicamente las variables independientes (producto, precio, plaza y promoción).

\section{Variable independiente: Producto}

Definición

conceptual:

Definición

operacional:

Dimensión

Atributos del producto étnico mexicano

Consumo étnico mexicano

Expresión de la cultura mexicana

Costumbres y

tradiciones

mexicanas

Es un conjunto de atributos tangibles e intangibles que abarcan; empaque, color, precio, calidad y marca, además del servicio y la reputación del vendedor, el producto puede ser un bien, un servicio, un lugar, una persona o una idea (Stanton, Etzel, \& Walker, 2012).

Está determinada por los atributos del producto étnico mexicano, el consumo étnico mexicano, la expresión de la cultura mexicana y las costumbres y tradiciones mexicanas.

\section{Indicador}

Ingredientes más representativos de la gastronomía mexicana: maíz, chile, y frijol.

La percepción del origen de los productos gastronómicos mexicanos a través de su marca. Influencia de compra en los consumidores nacionales. Reconocimiento mundial de la gastronomía mexicana.

Refleja la identidad de la cultura mexicana.

Adquirir parte la cultura mexicana a través de la costumbre del consumo de alimentos éticos.

Historia de México

Formas de preparación ítems

1.-El maíz, el chile y el frijol

identifican a la gastronomía

2.-Reconoce el origen de los

alimentos étnicos mexicanos a través de su marca.

54321

3.-Consume productos

54321

gastronómicos étnicos mexicanos

por lo menos una vez al mes.

4.-Le enorgullece consumir alimentos étnicos mexicanos.

5.-Los platillos mexicanos representan $\quad \begin{array}{lllll}5 & 4 & 3 & 2 & 1\end{array}$ totalmente la cultura mexicana.

6.-Al momento de consumir productos gastronómicos mexicanos adquiere parte de la cultura mexicana.

7.-Relaciona los alimentos étnicos

543221

Escala Likert

54321 mexicanos con parte de su historia.

8.-Al comprar productos étnicos mexicanos, valora su forma de preparación tradicional.

\section{Variable independiente: Precio}

Definición conceptual:

Es la suma de todos los valores que los consumidores entregan a cambio de los beneficios que obtienen por poseer o utilizar un producto o servicio (Kotler \& Armstrong, 2013).

Definición operacional: Está determinado por el poder adquisitivo, los precios internacionales y la elasticidad de la demanda.

Dimensión

Poder adquisitivo
Indicador

Capacidad de compra

Economía ítems

Escala Likert

9.-El precio de los productos gastronómicos mexicanos es accesible para su bolsillo. 10.-Comprar alimentos étnicos mexicanos reduce su economía. 
Precios

internacionales
La relación de un precio alto por ser un producto gastronómico mexicano importado. Relación del precio de los productos étnicos mexicanos con el posicionamiento de su marca.
11.-Está de acuerdo que un

54321 producto étnico mexicano importado deba tener un precio más alto que el de los productos nacionales.

12.-Considera que las marcas más posicionadas de los productos étnicos mexicanos deben tener un precio más alto que el de su competencia.

Elasticidad de la demanda

Variable independiente

Definición conceptual:

Definición operacional:

\section{Dimensión}

Puntos de venta

Disponibilidad de compra

Importación
Cantidad demandada de productos gastronómicos étnicos mexicanos.
13.-Sí de pronto los precios de los 54321 productos étnicos mexicanos incrementan los seguiría comprando.

\section{Plaza}

Es el conjunto de organizaciones interdependientes involucradas en el proceso de poner un producto o servicio para su uso o consumo por el consumidor o por otras empresas (Kotler \& Armstrong, 2013).

Está determinada por los puntos de venta, la disponibilidad de compra, la comunicación y la exportación.

Indicador
Suficientes puntos de venta
Apartados especiales para
alimentos étnicos mexicanos en
supermercados.

Accesibilidad de compra

Viabilidad de importación de productos étnicos mexicanos en España

ítems

Escala Likert

14.-Considera que existen suficientes 543321
puntos de venta de productos gastronómicos mexicanos en su localidad. 15.- Existen apartados especiales en los supermercados para los productos gastronómicos étnicos mexicanos. 16.-Los productos gastronómicos étnicos mexicanos son fáciles de conseguir en su localidad.

17.-Considera que es viable la importación de productos mexicanos en España, teniendo como punto de principal Madrid.

\section{Variable independiente: Promoción}

Definición conceptual:

Es el conjunto de herramientas de publicidad, promoción de ventas, relaciones públicas, venta personal y mercadotecnia directa, que utiliza una empresa con el fin de lograr sus objetivos de mercadotecnia y publicidad (Kotler \& Armstrong, 2013).

Definición operacional:

\section{Dimensión}

Publicidad

Está determinada por la publicidad, las técnicas de promoción de ventas y la comunicación.

Indicador

Publicidad de los productos

étnicos a través de la televisión

Publicidad de boca en boca

Promociones en la venta de

ítems

18.-Consume alimentos mexicanos

Escala Likert

porque los ha conocido a través de la televisión. 19.-Considera que ha conocido los platillos mexicanos por medio de comentarios de familiares y amigos. 
Técnicas de promoción

de ventas

Comunicación productos étnicos mexicanos.

Medios de comunicación
20.- Aprovecha las promociones

54321 existentes cuando compra alimentos mexicanos.

21.- Los medios de comunicación

543321 han sido un punto clave para el conocimiento y consumo de los productos gastronómicos étnicos mexicanos.

Fuente: Elaboración propia.

Para poder realizar la operacionalización de las variables de forma cuantitativa se requirió del siguiente tipo de escala Likert a su como la asignación de sus valores (Tabla 6).

\section{Tabla 6. Escala Likert y valores asignados que van del 1 al 5.}

\begin{tabular}{ccccc}
\hline Totalmente de acuerdo & De acuerdo & Neutral & En desacuerdo & Totalmente en desacuerdo \\
1 & 2 & 3 & 4 & 5 \\
\hline
\end{tabular}

Fuente: Elaboración propia.

Los valores de correlación de Pearson van de correlación negativa perfecta $(-0.91$ a -1.00) a correlación positiva perfecta $(+0.91$ a +1.00). La presentación de los resultados, así como de su interpretación y la comprobación de hipótesis, se presentan en la Tabla 7. Se muestran las cuatro hipótesis de trabajo establecidas que corresponden a las cuatro variables independientes producto, precio, plaza y promoción con sus respectivos valores en resultados, su interpre- tación de Pearson y comentarios. h1: producto, 0.352 de coeficiente de correlación de Pearson, significando una correlación positiva media.h2: producto, 0.107 de coeficiente de correlación de Pearson, significando una correlación positiva débil. h3: plaza, 0.314 de coeficiente de correlación de Pearson, significando una correlación positiva media. h4: promoción, 0.101 de coeficiente de correlación de Pearson, significando una correlación positiva débil.

Tabla 7. Interpretación de resultados.

\begin{tabular}{|c|c|c|}
\hline Hipótesis & Resultados & Comentarios \\
\hline H1: Producto & $\begin{array}{l}\text { El coeficiente de correlación } \\
\text { del producto momento de } \\
\text { Pearson (r) fue de } 0.352\end{array}$ & $\begin{array}{l}\text { La hipótesis sometida a prueba, se considera que aporta } \\
\text { evidencia a favor, siendo una variable directamente } \\
\text { proporcional con un significado de correlación } \\
\text { de Pearson positiva media. }\end{array}$ \\
\hline H2: Precio & $\begin{array}{l}\text { El coeficiente de correlación } \\
\text { del producto momento de } \\
\text { Pearson (r) fue de } 0.107\end{array}$ & $\begin{array}{l}\text { La hipótesis sometida a prueba, se considera } \\
\text { que aporta evidencia a favor, siendo una variable } \\
\text { directamente proporcional con un significado de } \\
\text { correlación de Pearson positiva débil. }\end{array}$ \\
\hline H3: Plaza & $\begin{array}{l}\text { El coeficiente de correlación } \\
\text { del producto momento de } \\
\text { Pearson (r) fue de } 0.314\end{array}$ & $\begin{array}{l}\text { La hipótesis sometida a prueba, se considera que aporta } \\
\text { evidencia a favor, siendo una variable directamente } \\
\text { proporcional con un significado de correlación de } \\
\text { Pearson positiva media. }\end{array}$ \\
\hline H4: Promoción & $\begin{array}{l}\text { El coeficiente de correlación } \\
\text { del producto momento de } \\
\text { Pearson (r) fue de } 0.101 .\end{array}$ & $\begin{array}{l}\text { La hipótesis sometida a prueba, se considera que aporta } \\
\text { evidencia a favor, siendo una variable directamente } \\
\text { proporcional con un significado de correlación de Pearson } \\
\text { positiva débil. }\end{array}$ \\
\hline
\end{tabular}

Fuente: Elaboración propia con base a metodología. 
En cuanto a la parte cualitativa, según la encuesta y entrevista a los 384 ciudadanos de Madrid con un rango de edad ente los 20 y 60 años de edad, arrojaron los siguientes datos descriptivos; 1 Lugar de procedencia (Madrid 357, Toledo 14, Guadalajara 7 y Barcelona 6), 2 Ocupación (estudiantes 62, empresarios 25, trabajadores de gobierno 84 , trabajadores en empresas 185 , otros 28) 3 Nivel de estudios (Bachillerato 174, grado 203 y máster 7) 4 Edad: rango de 20 a 30 (62), rango de 30 a 40 (157), rango de 40 a 50 (137), rango de 50 a 60 (28).

\section{CONCLUSIONES}

Después de haber realizado la investigación de campo y haber procesado la información obtenida estadísticamente a través del coeficiente de correlación de Pearson y haber obtenido los resultados, se concluye que se logra cumplir con el objetivo general y la comprobación de la hipótesis general aportando evidencia a favor al ser positivas y directamente proporcionales al consumo étnico de gastronomía mexicana en Madrid, España utilizando el modelo clásico de las 4P's (Producto, precio, plaza y promoción) de los autores Borden y Mc Carthy, como variables independientes y su relación con el consumo étnico de gastronomía mexicana en Madrid como variable dependiente correlacionando estas variables a través del coeficiente de correlación de Pearson.

Se muestra que las variables independientes; producto con un valor de 0.352 de coeficiente de correlación de Pearson y la variable independiente plaza con un valor de 0.314 de coeficiente de correlación de Pearson, son las que más relación e influencia tuvieron con una correlación positiva media, seguidas de las variables independientes precio con un valor de 0.107 de coeficiente de correlación de Pearson y promoción con un valor de 0.101 de coeficiente de correlación de Pearson con una correlación positiva débil. A manera de resumen se demuestra que estas cuatro variables oscilan de manera positiva entre los rangos medio y débil.

Por último, la investigación fue original y especifica apor- tando al conocimiento teórico conceptos sobre gastronomía mexicana, producto étnico, los cuales evidencian a favor la teoría hacia el consumo de los productos étnicos.

\section{REFERENCIAS}

National Geographic. (12 de Febrero de 2015). Obtenido de http://www.nationalgeographic.com.es/ viajes/grandes-reportajes/los-10-mejores-destinos-gastronomicos-del-mundo-2 8947/1

Alva, C. I. (2012). Historia de la gastronomía. México: RED TERCER MILENIO. Obtenido de http://www. aliat.org.mx/BibliotecasDigitales/economico administrativo/Historia de la gastronomia.pdf

Alvarado, L. (2011). Obtenido de http://gastronomia890611kp11/15010614.blogspot.mx/p/tipos-de-gastronomia.html

Aparicio, D. (07 de 04 de 2014). 20 minutos. Obtenido de http://www.20minutos.es/noticia/2102700/0/ gastronomia/patrimonio-inmaterial-de-la-humanidad/dieta-mediterranea/

Araujo, G. (2007). Monografías.com. Obtenido de http:// www.monografias.com/trabajos12/elproduc/elproduc.shtml

Camarena , D., \& Sanjuán, A. (Noviembre de 2007). El mercado de comida étnica en España: el caso de la comida mexicana. Obtenido de http://www.scielo. org.mx/pdf/estsoc/v16n31/v16n31a1.pdf

CEPAL. (Abril de 2004). Obtenido de https://www.cepal.org/es/publicaciones/4931-pequenas-empresas-productos-etnicos-nostalgia-oportunidades-mercado-internacional

Chávez. (23 de mayo de 2014). El Financiero. Obtenido de 10 productos estrella de las exportaciones mexicanas: http://www.elfinanciero.com.mx/economia/10-productos-estrella-de-las-exportaciones-mexicanas.html

CNN. (16 de septiembre de 2018). Los 6 productos mexicanos más solicitados en el extranjero. Obtenido de https://cnnespanol.cnn.com/2017/09/16/ los-6-productos-mexicanos-mas-solicitados-en-el-extranjero/\#0 
Compartido - Fideicomiso de Riesgo. (13 de Septiembre de 2017). Gob.mx. Obtenido de El maíz, base de la dieta mexicana desde época prehispánica: https://www.gob.mx/firco/articulos/ el-maiz-base-de-la-dieta-mexicana-desde-epoca-prehispanica

Constantinides, E. (2006). The Marketing Mix Revisited: Towards the 21st Century Marketing. Journal of Marketing Management, 407-438. doi: https://doi. org/10.1362/026725706776861190

Cruz, M. (5 de Julio de 2017). ELMUNDO. Obtenido de http://www.elmundo.es/espana/2017/07/05/ 595d076de2704ede7e8b45f5.html

Cruz, M., López Cerdán , C., \& Schatan , C. (Abril de 2004). Pequeñas empresas, productos étnicos y de nostalgia: oportunidades en el mercado internacional. Obtenido de https://www.cepal.org/publicaciones/xml/7/14737/L589-1.pdf

EL FINANCIERO. (23 de MAYO de 2014). Economia. Obtenido de 10 productos estrella de las exportaciones mexicanas: http://www.elfinanciero.com.mx/ economia/10-productos-estrella-de-las-exportaciones-mexicanas

Fernández, E. (10 de Diciembre de 2016). FORBES. Obtenido de https://www.forbes.com.mx/forbes-life/ gastronomia-mexicana-patrimonio-de-la-humanidad/

FORBES. (11 de Julio de 2014). Obtenido de https://www. forbes.com.mx/gastronomia-mexicana-la-conquista-de-europa/

Frías, D. (2014). APUNTES DE SPSS. Obtenido de Universidad de Valencia: https://www.uv.es/friasnav/ ApuntesSPSS.pdf

Giménez, G. (2007). Estudios sobre la cultura y las identidades sociales. CONACULTA \& ITESO.

Hinojosa, M. d. (29 de Abril de 2011). Wordpress. Obtenido de https://esdaiposgrados.wordpress. com/2011/04/29/mexico-y-sus-regiones-gastronomicas/

Kotler, P., \& Armstrong, G. (2013). Fundamentos de Marketing. México: Pearson .

LA VANGUARDIA. (22 de 06 de 2017). Obtenido de http://www.lavanguardia.com/ vida/20170622/423600390043/espana-es-elmejor-pais-para-nacer-por-su-alto-nivel-de-bienestar-y-salud-segun-un-ranking-de-deloitte-y-spi. html

Mondragón, A. (23 de 3 de 2015). EL FINANCIERO . Obtenido de http://www.elfinanciero.com.mx/suplementos/la-gastronomia-mexicana-es-reconocida-en-todo-el-mundo.html

Montanari, M. (2006). La comida como cultura. España: Ediciones Trea.

Movimiento Cientifico. (2014). ARTículos de ReflexiónMovimientoCientífico98Mov.cient.Vol.8 (1): 98104ISSN: 2011-7191. Enero a Diciembre de 2014USO DE LA CORRELACIÓN DE SPEARMAN EN UN ESTUDIO DE INTERVENCIÓN EN FISIOTERAPIA. Movimiento Cientifico, 8(1), 98-104.

Muzeum Gastronomie. (14 de Marzo de 2018). La Gastronomía . Obtenido de Citas Famosas Sobre Gastronomia Chefs y Comida: http://www.muzeumgastronomie.cz/es/node/75

OMT. (Mayo de 2016). Obtenido de http://www.siimt. $\mathrm{com} /$ work/models/siimt/Resource/1de0bb9d -8199-48fb-af9a-769464f99875/PDF RankingOMT_2015_May16.pdf

Ramos, M. (2011). La construcción teórica de la gastronomía como producto turístico cultural en México. Obtenido de http://egourmet.com.mx/sociales. php?id social $=493$

Skyscanner. (6 de Julio de 2016). Obtenido de https:// www.skyscanner.es/noticias/10-razones-por-lasque-espana-es-el-mejor-pais-del-mundo-na

Stanton, W. J., Etzel, M. J., \& Walker, B. J. (2007). Fundamentos de Marketing. México: McGraw-Hill.

Stanton, W., Etzel, M., \& Walker, B. (2012). Fundamentos de Marketing. México: Mc Graw-Hill.

Torres, G., Madrid de Zito Fontán, L., \& Santoni , E. (2004). El alimento, la cocina étnica, la gastronomía nacional. Elemento patrimonial y un referente de la. REDALYC, 61.

UNESCO. (2017). Organización de las Naciones Unidas para la Educación, la Ciencia y la Cultura. Obtenido de http://www.unesco.org/new/es/mexico/workareas/culture/intangible-heritage/ 